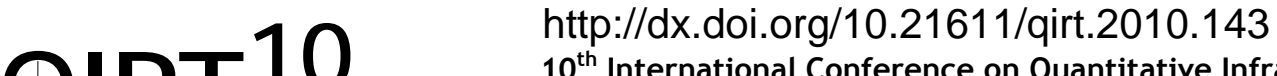 \\ $10^{\text {th }}$ International Conference on Quantitative InfraRed Thermography \\ July 27-30, 2010, Québec (Canada)
}

\section{Imaging and quantitative characterization of bilateral vasomotor reactions in humans using high-performance thermography}

\author{
by Boris G. Vainer* and Arcady L. Markel**
}

* Rzhanov Institute of Semiconductor Physics, Russian Academy of Sciences, Siberian Branch, Novosibirsk, Russia

** Institute of Cytology and Genetics, Russian Academy of Sciences, Siberian Branch, Novosibirsk, Russia

e-mail: BGV@isp.nsc.ru

\begin{abstract}
We demonstrate that the use of high-performance FPA-based infrared thermography combined with an unilateral impact on the organism is a powerful new approach toward registration and understanding the diversity of bilateral vasomotor reactions in humans. This work provides strong evidence that unilateral arterial occlusion and post-occlusive reactive hyperemia provokes such a reaction. The importance of the described IRT-based method to medicine is indubitable because reactive hyperemia characterizes ability of the organism to maintain peripheral circulation after a sudden decrease in perfusion pressure (due to, for instance, thrombosis or embolization). Different vasomotor reactions in humans revealed in our work permits to discriminate population between the receptive and the immune to remote ischemic preconditioning.
\end{abstract}

Vascular reactivity (VR) refers to changes in the blood vessels in response to internal or external stimuli. Quantitative characterization of VR can bring important information about the state of cardiovascular system. The well-known phenomenon associated with VR is reactive hyperemia $(\mathrm{RH})$. This phenomenon has been amply dealt with ${ }^{1-3}$. $R H$ is a complex vascular reaction that occurs after a period of tissue ischemia. It primarily depends on both local production of tissue substances entailing the post-ischemic vascular relaxation and autonomous nervous system response to these local processes. Recent studies in humans have shown that some endothelium-derived factors contribute to $\mathrm{RH}$, and the peak of brachial artery hyperemic flow registered after temporary brachial cuff assisted occlusion relates inversely to the risk of traditional cardiovascular disease. Endothelial dysfunction is an attendant feature of established atherosclerosis (AS). It is also a forerunner of AS and a sign manifesting about AS susceptibility.

There are many varieties of methods elaborated for the investigation of regional blood flow, microcirculation, and $\mathrm{RH}$ : echo-planar imaging, high-resolution ultrasound, near-infrared spectroscopy (NIRS), radionuclide plethysmography, strain gauge plethysmography, capillaroscopy, laser Doppler flowmetry (LDF), digital thermal monitoring (DTM), etc. None of these methods can be accepted as ideal technique. Some of them are too complicated, uncomfortable or cumbersome for widespread use. Some techniques (LDF, NIRS, DTM ${ }^{3}$, etc.) allow only local skin regions to be analyzed. Although vascular imaging and biochemical markers demonstrate at times a considerable promise, assessment of vascular function remains of particular interest.

The methods applying the principles of the computer-equipped focal-plane array (FPA) based high-performance infrared thermography (IRT) are today's novel technologies in quantitative biomedical research. They make possible to reveal basically new information about function of the circulatory system ${ }^{4}$. The main advantage of IRT is the coverage of spacious body area concurrently underwent an examination. In the present work, high-performance IRT allowed us to reveal and clearly demonstrate some inner processes hidden from view inside the human organism. In particular, on the strength of our experimental data we concluded that real evaluation of the true degree of $\mathrm{RH}$ originated from endothelium-derived factors necessitates differentiating this hyperemia from that originated from nervous system conditioned vascular smooth muscle reflex; using the IRT method, we observed and quantitatively represented both above-mentioned manifestations of vascular response.

Thirty five predominantly healthy volunteers were studied. Time dependence of RH was investigated in the majority of cases after 1.5-3 min occlusion resulted from the brachial cuff rapid inflating to a pressure of $200-250 \mathrm{mmHg}$. Blood pressure cuff was taken from the standard A\&D Compact Desktop Monitor, Model UA-605. Although the cuff was applied to one of the shoulders, thermal patterns of both hands simultaneously were registered and saved in real time. 2D thermograms as well as the time dependence of temperature distantly measured in some selected points on skin were recorded with the use of high-sensitive $(0.03 \mathrm{~K})$ and quick-operating (up to $100 \mathrm{fps}$ ) FPA-based infrared camera TKVr-IFP fixed over the hands.

We revealed and analyzed several types of vasomotor reactions in investigated subjects. One of the reactions is exhibited in figures 1 and 2. During occlusion, the temperature decrease in ischemic hand was normally recorded. After cuff deflation, the compressed hand temperature was increased in a time dependent manner (reactive hyperemia). Quite similar and synchronous skin temperature dynamics was normally observed in the vacant hand. Remarkably, not only the postischemic temperature increase was detected in vacant hand, but also the temperature fall during cuff inflation just like in the compressed hand. The above-mentioned bilateral effect consisting in the both hands synchronous change (decrease and 
increase) in temperature after unilateral impact was discovered recently with the use of FPA-based IRT and demonstrated in [5].

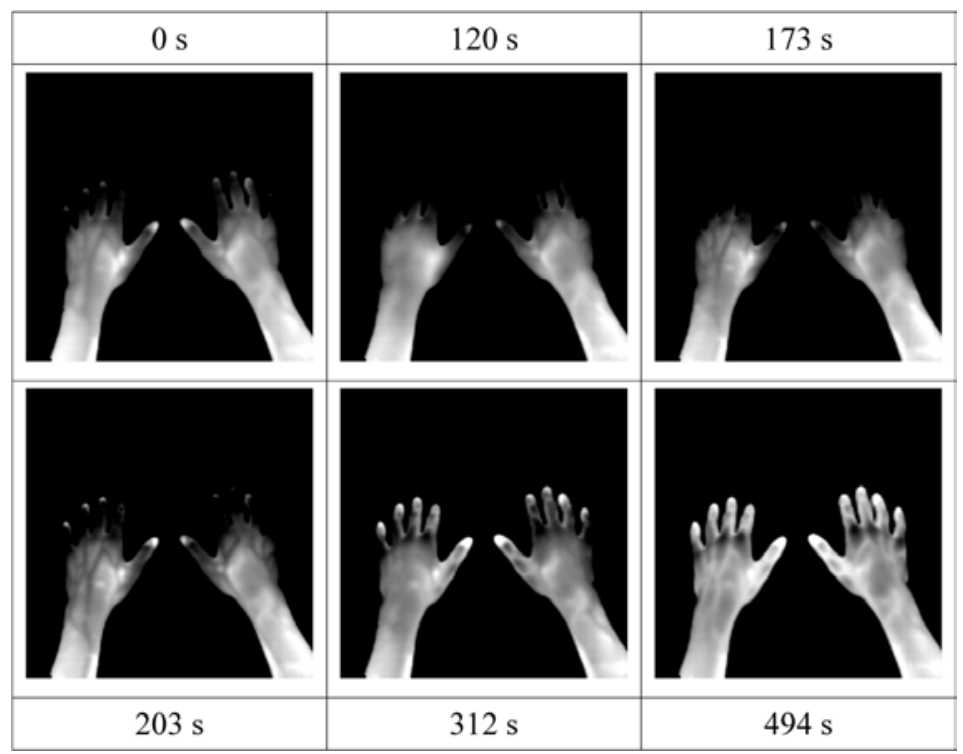

Fig. 1. Thermal patterns of upper extremities displaying synchronous bilateral vasomotor reaction during and after mechanical unilateral occlusion of blood flow. Shown is the symmetric thermal behavior of both hands in response to occlusion of blood flow in left hand only. Dark- and light-color thermal representations of superficial veins correspond to cold and warm blood drainage from cooled (at 2-min occlusion) and warmed (in the course of the left hand $\mathrm{RH}$ ) fingers, respectively. The legend near each thermogram is the time interval $\Delta t$ elapsed since the occlusion is started (occlusion is finished at $\Delta t=120 \mathrm{~s}$ ).

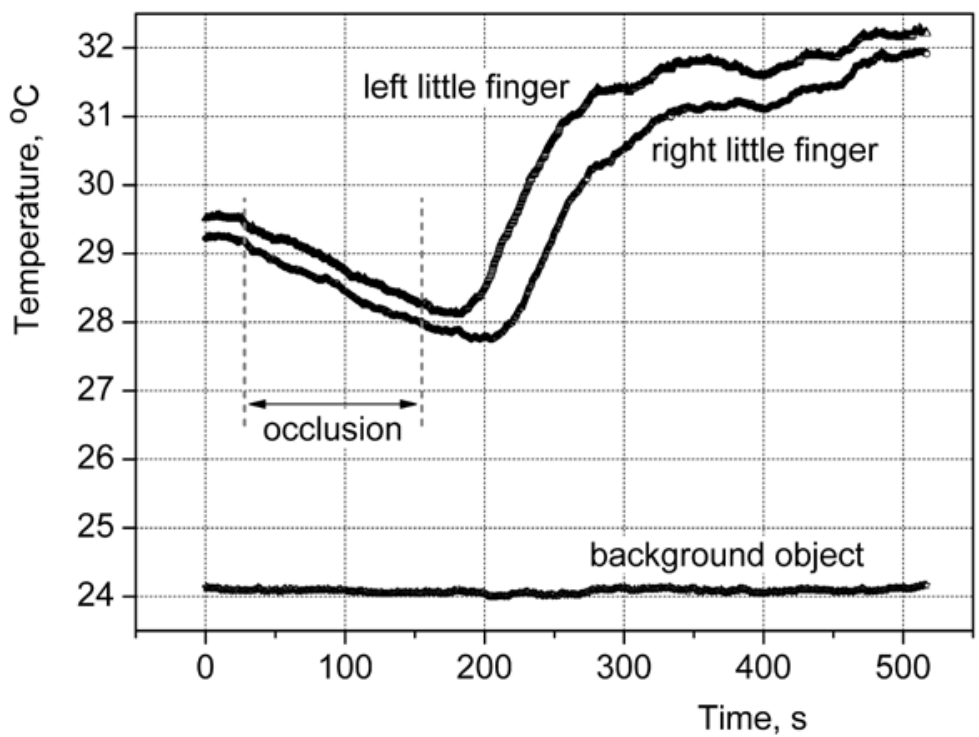

Fig. 2. Time dependence of cutaneous temperature determined from $2 D$ digital thermograms during arterial occlusion and after deflation of cuff wrapped around left upper arm. Two upper curves are plotted for the areas occupied by left and right little fingers. Lower curve demonstrates that the temperature of a reference (background) object located within the "field of visibility" of IR camera remains virtually unchanged. 

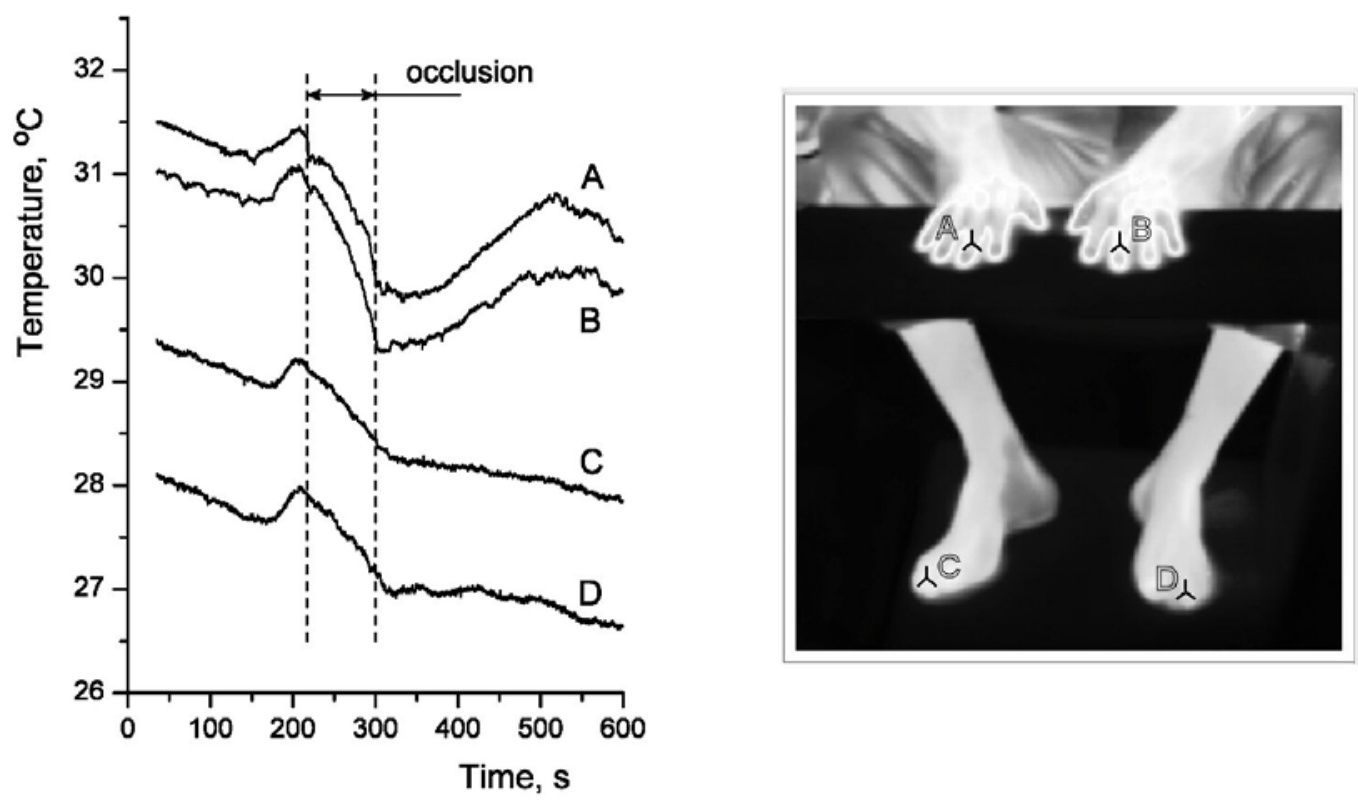

Fig. 3. Time dependence of cutaneous temperature determined at four check points shown in the right picture during arterial occlusion and after deflation of cuff wrapped around right upper arm.

It should be noted that the obtained by IRT in our work typical vacant-hand temperature profile differs from that obtained by DTM and exhibited in [3].

In seven healthy subjects, we monitored vasomotor reaction of both upper and lower extremities simultaneously. Thermal manifestation in lower extremities was normally akin to that in the right hand, even if lower intensity (figure 3). At the same time, asynchronous behavior of compressed and vacant extremities as well as a hardly perceptible reaction of them to occlusion were also observed. It was suggested that the changes in vacant upper extremity and in lower extremities were induced by autonomous nervous reflexes stimulated by the compressed hand brief ischemic state and post-ischemic vascular relaxation. Occurrence of different vasomotor reactions we revealed in humans permits to discriminate population between the receptive and the immune to remote ischemic preconditioning [6]. IRT can serve for this discrimination as the most convenient and informative instrument.

Thus infrared monitoring and quantitative analysis of bilateral vasomotor reactions in humans give IRT the opportunity to compare the direct (mainly endothelium dependent) mechanisms of post ischemic vascular relaxation from indirect (reflectory induced) ones. The extremity blood inflow transient occlusion as experimental therapeutic intervention combined with FPA-based high-performance IRT monitoring allows disclosing the specific behavior of neurovascular system and $\mathrm{RH}$ in humans and making clearer its nature. Such a testing might have clinical utility for patient management. The results of this work prove IRT as a promising method for cardiovascular diagnostics and physiological research.

\section{Acknowledgments}

We thank all volunteers for their taking part in the present study. This work was supported by Siberian Branch of the Russian Academy of Sciences (Integration Project grant 91-2009).

\section{REFERENCES}

[1] Imms F.J., Lee W.-S., Ludlow P.G. Reactive hyperaemia in the human forearm, Q. J. Exp. Physiol., 73, p. 203-215, 1988. 
[2] Lorenzo S., Minson C.T. Human cutaneous reactive hyperaemia: role of BKCa channels and sensory nerves, J. Physiol., 585, p. 295-303, 2007.

[3] Ahmadi N., Nabavi V., Nuguri V., Hajsadeghi F., Flores F., Akhtar M., Kleis S., Hecht H., Naghavi M., Budoff M. Low fingertip temperature rebound measured by digital thermal monitoring strongly correlates with the presence and extent of coronary artery disease diagnosed by 64-slice multi-detector computed tomography. Int. J. Cardiovasc. Imaging, 25, p. 725-738, 2009.

[4] Vainer B.G. FPA-based infrared thermography as applied to the study of cutaneous perspiration and stimulated vascular response in humans, Physics in Medicine and Biology, 50, p. R63-R94, 2005.

[5] Vainer B.G. The use of infrared thermography for the investigation of thermoregulation in humans. - Ch. 5 in: Body temperature regulation / Austin B. Cisneros and Bryan L. Goins, Editors, Nova Science Publishers, Inc., New York, 360 p., 2009.

[6] Kharbanda R.K., Mortensen U.M., White P.A., Kristiansen S.B., Schmidt M.R., Hoschtitzky J.A., Vogel M., Sorensen K., Redington A.N., MacAllister R. Transient limb ischemia induces remote ischemic preconditioning in vivo, Circulation, 106, p. 2881-2883, 2002. 